Chapman University

Chapman University Digital Commons

Communication Faculty Articles and Research

School of Communication

6-22-2019

\title{
A Meta-Analytic Examination of the Continued Influence of Misinformation in the Face of Correction: How Powerful Is It, Why Does It Happen, and How to Stop It?
}

Nathan Walter

Northwestern University

Riva Tukachinsky

Chapman University, tukachin@chapman.edu

Follow this and additional works at: https://digitalcommons.chapman.edu/comm_articles

Part of the Communication Technology and New Media Commons, Critical and Cultural Studies Commons, Mass Communication Commons, and the Other Communication Commons

\section{Recommended Citation}

Walter, N., \& Tukachinsky, R. (2019). A meta-analytic examination of the continued influence of misinformation in the face of correction: How powerful is it, why does it happen, and how to stop it? Communication Research. https://doi.org/10.1177/ 0093650219854600 


\section{A Meta-Analytic Examination of the Continued Influence of Misinformation in the Face of Correction: How Powerful Is It, Why Does It Happen, and How to Stop It?}

\section{Comments}

This is an Accepted Manuscript of an article published in Communication Research in 2019, available online at DOI: $10.1177 / 0093650219854600$. It may differ slightly from the final version of record.

\section{Copyright}

Taylor \& Francis 


\begin{abstract}
A meta-analysis was conducted to examine the extent of continued influence of misinformation in the face of correction and the theoretical explanations of this phenomenon. Aggregation of results from 32 studies $(N=6,527)$ revealed that, on average, correction does not entirely eliminate the effect of misinformation $(r=-.05, p=.045)$. Corrective messages were found to be more successful when they are coherent, consistent with the audience's world-view, and delivered by the source of the misinformation itself. Corrections are less effective if the misinformation was attributed to a credible source, the misinformation has been repeated multiple times prior to correction, or when there was a time lag between the delivery of the misinformation and the correction. These findings are consistent with predictions based on theories of mental models and offer concrete recommendations for practitioners.
\end{abstract}

Keywords: Misinformation; correction; continued influence; meta-analysis 


\section{A Meta-Analytic Examination of The Continued Influence of Misinformation in the Face of Correction: How Powerful is it, why does it Happen, and how to Stop it?}

In the contemporary media environment individuals often encounter myths, rumors, reporting errors, and misinformation deliberately or inadvertently circulated by the media, governments, and other interest groups. Recent research shows that on social media, false news

reach more people and spread more rapidly than accurate news (Vosoughi, Roy, \& Aral, 2018). For example, during the 2016 presidential elections, nearly one in four Americans was exposed to a false news story but fact-checking stories reached only a narrow set of media consumers (Guess, Nyhan \& Reifler, 2018). This spread of misinformation poses a challenge to traditional news. For instance, the CNN Tonight anchor, Don Lemon, expressed his frustration, saying on the air: "I spend most of my time trying to debunk falsities and lies” (Lemon, April 2018).

Although massive efforts are devoted to debunking rumors, correcting of misinformation, and fact-checking, scientific evidence of the effectiveness of these measures appears to be inconsistent (Walter \& Murphy, 2018). While some studies have found corrective information to reduce the effect of falsehoods (e.g., Skurnik, Yoon, \& Schwarz, 2005) other studies suggest that individuals may struggle to discount misinformation (e.g., Schwarz et al., 2007). In fact, corrective messages can even backfire by increasing support for the discredited position (Nyhan \& Reifler, 2010). Multiple theoretical frameworks have been developed in an attempt to account for the complexities of such continued effect of misinformation (Lewandowsky, Ecker, Seifert, Schwarz, \& Cook, 2012). However, individual studies that examine those theoretical propositions are typically designed to test a single explanatory mechanism at a time, whereas in reality, multiple psychological processes are likely to operate simultaneously. Moreover, when examined in isolation, individual studies do not permit valid generalizations, as each individual 
study is limited to a particular set of messages, samples, and measurements (O’Keefe, 2015).

The present study employs a meta-analytical approach to advance the understanding of the scope and mechanisms underlying the continued influence of misinformation in the face of correction by aggregating results across multiple studies. Once the overall effect size is determined, a series of moderation analyses test a number of previously proposed explanations to the continued effect of misinformation. Specifically, the study investigates: (a) characteristics of misinformation that make the misinformation harder to root out and (b) characteristics that make certain forms of correction more or less effective. By systematically exploring the properties of the misinformation and the characteristics of corrective information, the current study advances the theoretical understanding of the psychology of managing conflicting information and offers practical guidelines for designing more effective correction messages.

\section{The Continued Influence of Misinformation}

The continued influence of misinformation can be thought of as one of many information processing biases commonly exhibited by media consumers. For instance, it is well documented that individuals privilege exemplar-based information over base-rate information, such that individuals' perceptions of an issue can be skewed by unrepresentative exemplars (Allen, Preiss, \& Gayle, 2006; Zillmann, 2002). Importantly, whereas the exemplification theory is concerned with how individuals manage different types of information (statistics vs. exemplars) that are both correct, the current study explores responses to correction or retraction of the same type of information such that the status of the initial communication changes from true to false.

Past meta-analyses examining the effect of correction compared to uncorrected misinformation control conditions have found that corrective messages can, indeed, significantly reduce belief in misinformation (Blank \& Launay, 2014; Walter \& Murphy, 2018). These 
findings importantly demonstrate that exposure to a correction is better than receiving no correction at all. However, this choice of control largely overlooks the role played by misinformation. Recently, Chan, Jones, Hall Jamieson and Albarracín (2017) aggregated the results of eight reports to reveal a large effect $(d=.75-1.06)$ of misinformation in the face of correction. However, given the limited scope of the study (eight reports published from 1994 to 2015 and three moderators) and its exploratory nature, there is a need for a more complete and theory-driven synthesis of the literature.

To further advance this field, the current meta-analysis specifically examines randomized experiments comparing between attitudes and beliefs of individuals who were exposed to corrected misinformation and those who were not exposed to misinformation in the first place. This contrast allows to determine the extent to which correction interventions revert the individuals’ attitudes and beliefs back to baseline (Ecker, Lewandowsky, Fenton, \& Martin, 2014). Given that participants are randomly assigned to conditions, they are expected to be equivalent in terms of their baseline attitudes and other characteristics. Ideally, if corrective messages were fully effective, there would be no significant difference between the beliefs of those exposed to the correction following a false message compared to those who were never exposed to the misinformation in the first place. Thus, significant differences between these two conditions represent the continued effect of misinformation in spite of the correction. The first aim of the present study is to identify the average continued effect of misinformation:

RQ1: What is the effect of correction of misinformation compared to a nomisinformation control?

Once the average effect size of the continued misinformation influence is identified, theoretical mechanisms underlying this effect are explored. 


\section{Theoretical Explanations of the Continued Influence of Misinformation}

Multiple theories explicate the effect of misinformation in spite of correction. These are by no means competing theories. Rather they complement each other and at times may overlap.

Mental models. According to this approach, individuals construct a mental representation of unfolding events but seem reluctant to dismiss key information if an alternative explanation was sufficient to fill this void (Ecker, Lewandowky, Swire, \& Chang, 2011). In other words, individuals may prefer to uphold a coherent, albeit incorrect mental model than to be left with an incomplete one (Johnson \& Seifert, 1994).

Hence, to be effective, a correction should offer information that can successfully replace the refuted components of the mental model without compromising the coherence of all the existing elements in the story (Lewandowsky et al., 2012). Rather than merely discounting the misinformation, a successful correction should provide an alternative explanation, offering evidence, and/or explaining why the misinformation was presented in the first place. For instance, in the context of vaccine safety, if people think that health organizations take part in an elaborate conspiracy to conceal relevant information from concerned parents, a message from health experts negating the vaccination-autism link will do little to reduce belief in misinformation. In fact, such efforts may backfire by making the audiences cling even harder to anti-vaccination myths. A more effective correction would try to substitute the original mental model with a coherent explanation that includes information about vaccine safety as well as explain the corrupt history of the anti-vaccine movement. In sum, the richness of detail in the original misinformation and the extent to which the correction offers a coherent explanatory framework should determine the continued influence effect (Cappella, Maloney, Ophir, \& Brennan, 2015). It is hypothesized that: 
H1a: The continued influence of misinformation will be stronger for high compared to low-coherence misinformation.

H1b: The continued influence of misinformation will be stronger for low compared to high-coherence correction.

H1c: Misinformation and correction coherence will interact such that the continued influence effect will be stronger when misinformation coherence is high but correction coherence is low.

Once a mental model is constructed, it has been speculated that the longer the misinformation is held the more it becomes integrated into memory and is then more difficult to root out (Ecker, Lewandowsky, Cheung, \& Maybery, 2015). Ostensibly, although even immediately retracted information can have a trace effect, real-time corrections are easier to integrate into the mental model, potentially minimizing the effect of misinformation. Indeed, educational research consistently finds that immediate correction of an error is superior to a delayed correction (Barbetta, et al., 1994). Thus, the duration of time between exposure to the misinformation and the correction could also moderate the effect of misinformation:

$\mathrm{H} 2$ : The longer is the time delay between exposure to misinformation and its correction, the stronger is the continued influence of misinformation.

Fluency and familiarity. Familiarity and fluency serve as important heuristics in favoring certain information (Berinsky, 2015). Fluency refers to the metacognitive experience of the relative ease or difficulty of the thought process. Information can be fluent (i.e., highly accessible), or it can be disfluent - hard to understand and retrieved only through an effortful process (Schwarz, 2015). Overall, the literature suggests that the metacognitive experience of fluency guides truth judgments, such that fluent information is regarded as more truthful (Reber 
\& Schwarz, 1999).

In addition to the previously-discussed coherence, several factors can contribute to the sense of fluency, including visual elements (making statements appear easy or difficult to read) and the repetition of information. Repetition strengthens the experience of fluency and makes information more readily accessible for retrieval, more familiar, and more consequential in subsequent attitude formation (e.g., Hansen \& Wänke, 2009). Along with fluency, numerous judgment biases such as pluralistic ignorance (Lewandowsky et al., 2012) and perceived social consensus (Festinger, 1954) can potentially explain the ability of repetition to solidify belief in misinformation. As demonstrated in the context of eyewitness research, repetition, but not number of sources, increase susceptibility to misinformation (Foster, Huthwaite, Yesberg, Garry, \& Loftus, 2012). Remarkably, Berinsky (2015) argues that simply asking subjects to repeat a rumor to themselves - without any indication that the rumor is true - increases its effect that persist for weeks after the initial exposure. Thus, repetition of the misinformation is likely to make it take root further in the message recipients' minds. In fact, the correction itself can backfire by repeating misinformation. Since familiarity serves as a heuristic, corrective messages that repeat the misinformation attempting to quash inaccuracies through direct refutation can inadvertently facilitate misinformation fluency and make it even more impactful (Berinsky, 2015).

Following the same logic, it is suggested that repetition of the corrective message can strengthen its encoding, resulting in perceived familiarity and greater acceptance of the correction. Though only a limited number of studies systematically manipulated repetition of corrective information, there is some evidence that repeating a correction can reduce reliance on misinformation (Ecker, et al., 2011; Ozturk, Li, \& Sakamoto, 2015). It is hypothesized that: 
H3a: The continued influence of misinformation will be stronger when misinformation is repeated.

H3b: The continued influence of misinformation will be stronger when corrective messages repeat the misinformation compared to corrective messages that do not repeat the misinformation.

H3c: The continued influence of misinformation will be weaker when corrective information is repeated.

Worldview consistency. To reconcile preexisting beliefs with a seemingly contradicting reality, people are motivated to discount information that challenges their worldview by derogating the source of the message or questioning the accuracy of the claims (Kunda, 1990). In contrast, information that fits within people's belief system is often judged as probative and accepted at face value even when its validity is called into question by experts and authority figures. Motivated processing of information can result from: (a) an attitude congruency bias or (b) a disconfirmation bias. While the former bias focuses on scenarios where people provide more favorable evaluations of messages that support their prior beliefs, the latter deals with situations where disconfirming arguments are scrutinized and called into question, irrespective of their actual merit (Taber \& Lodge, 2006). In short, both biases suggest that when motivated to reach a certain conclusion, people can vary their level of attention and scrutiny of information to support their preferences (Chang, 2015).

Unsurprisingly, when information that is consistent with one's worldview is retracted, individuals are more likely to engage in motivated processing and discount the retraction even in the face of concrete evidence and mounting facts (Flynn, Nyhan, \& Reifler, 2017). Thus, it seems that the continued influence effect grows stronger when it aligns with partisan goals and, 
by the same token, corrections are more effective if they affirm people’s worldview.

Accordingly, it is hypothesized that:

H4: Counter-attitudinal corrections will lead to a stronger continued influence effect, compared with pro-attitudinal corrections ${ }^{1}$.

To further tap into the notion of worldview, the current meta-analysis considers the topic of the messages as a proxy-variable. Some studies on the continued influence of misinformation use real-life controversies (e.g., vaccination) as a context. In such cases, study participants are likely to have been exposed to the issue in the past and already developed a position.

Conversely, other studies construct scenarios that individuals will likely have no prior beliefs about (e.g., fictitious news about a fire). Such “constructed” and "real-life” topics should vary in the extent to which worldview plays a role in their processing (Lewandowsky et al., 2012). It is hypothesized that:

H5: The continued effect of misinformation will be stronger in real world contexts versus constructed contexts.

Source evaluation. Over half a century of research reveals that with few exceptions, credibility enhances persuasion outcomes (for review see Pornpitakpan, 2004). In particular, when lacking sufficient motivation and expertise, source credibility serves as a useful heuristic, guiding acceptance of the persuasive message (Petty \& Cacioppo, 1986). Similarly, in the misinformation literature, studies have found that messages are more effective when they are associated with a high-credibility source compared to a low-credibility source (Guillory \& Geraci, 2013). Though it is difficult to determine what lends credibility to a source of

\footnotetext{
${ }^{1}$ No hypotheses concerning counter-attitudinal misinformation and interaction between misinformation and correction were posed because of the inherent interdependency between the two. If the misinformation was attitudeconsistent, the correction is typically counter-attitudinal and vice versa.
} 
information (Lewandowsky et al., 2012), there is a general agreement that communicators’ credibility has a positive effect on both acceptance of misinformation and its correction (but see Cho, Martens, Kim, \& Rodrigue, 2011). Thus, it is hypothesized that:

H6a: The continued effect of misinformation will be stronger when the misinformation is attributed to a high-credibility source (compared to lowcredibility).

H6b: The continued effect of misinformation will be stronger when the correction is attributed to a low-credibility source (compared to high-credibility).

Another important dimension of the source involves equivalence between the source of the misinformation and the source of the correction. Admitting a mistake is critical for building trust (e.g., Rushton, Reina, \& Reina, 2007); thus, a corrective message retracting one’s previous statement should infuse confidence and acceptance of that message. Moreover, when a given source corrects their own prior communication, the correction would be seen as more trustworthy than a correction issued by a different party with possible ulterior motivations. Based on this logic, the Federal Trade Commission (FTC) requires that corrective advertising will be made by the same brand that was responsible for spreading the false information. For example, Doan’s analgesic products (Mazis \& Adkinson, 1976) and Warner-Lambert's Listerine mouthwash (Armstrong, Gurol, \& Russ, 1979) were tasked to sponsor and deliver campaigns to counteract their own past misleading advertising efforts. It is therefore hypothesized that:

H7: The continued effect of misinformation will be stronger when the source of the correction is different from (rather than equivalent to) the source of misinformation.

Negativity bias. Compared to positive information, negative information is more salient and is more rapidly recognized. Moreover, negative information is assigned greater weight and it 
is, therefore, more consequential in decision making and impression formation (for review see Baumeister, Bratslavsky, Finkenauer, \& Vohs, 2001; Ito, Larsen, Smith, \& Cacioppo, 1998; Rozin, \& Royzman, 2001). Unsurprisingly, then, negativity bias plays a role in transmission of information, as individuals are more likely to pass along information about negative events than about positive events when recounting a story containing both elements (Bebbington, MacLeod, Ellison, \& Fay, 2017). It is conceivable that the appeal of some misconceptions circulated in the media stems from its negative valance. The MMR vaccination-autism myth, conspiracy theories, and rumors of a scandal can be more salient, memorable, and compelling than the nonsensational, valance-neutral truths that attempt to debunk these false claims. In other words, negativity bias might, at least in part, be responsible for the persistent influence of misinformation when individuals continue to retain and retrieve refuted information: H8a: The continued effect of misinformation will be stronger when the misinformation is negative (vs. positive or neutral).

H8b: The continued effect of misinformation will be stronger when the correction is positive or neutral (vs. negative).

Retrieval failure due to negation. Lewandowsky et al. (2012) suggest that another challenge for discounting misinformation is failure in the processes of encoding and retrieval of memories. Specifically, according to the schema plus tag theory (Clark \& Chase, 1972), people encode negative memories by creating a positive memory with a negative tag. In other words, when the original misinformation is linguistically negated (e.g., "Jim is not guilty”) the information is encoded by adding a negative tag to an existing mental representation of the misinformation (Jim guilty - not). This tag can then be "lost" or fail to be subsequently retrieved. Thus, merely thinking of negation statements ("X is not Y") creates false memories of 
the negated object (Fiedler et al. 1996). To avoid this, affirming language (e.g., "John is innocent”) should be used in lieu of negating language (Mayo et al., 2014). In fact, the deleterious influence of correcting by negating, prompted a succinct recommendation to "avoid negations” (Nyhan \& Reifler, 2012, p. 1). Following this line of reasoning, it is hypothesized that:

H9: The continued influence of misinformation will be stronger when the correction message involves linguistic negation compared to affirmative language.

\section{Method}

\section{Sampling}

Literature search. Studies were located through a search in electronic databases (e.g., Google Scholar, JSTOR, PsychINFO, ProQuest, Communication and Mass Media Complete), using the search terms: debunking, misinformation, correction, “continued influence”, and their derivations. Then, the reference lists of these publications were examined to identify any prospective studies that were not obtained through the database search. Finally, 12 experts from the fields of communication, political science, cognitive and social psychology were contacted in order to solicit unpublished data and identify omissions in our sample.

Inclusion criteria. The goal of the present study was to identify the extent to which corrections can revert participants' state back to baseline. Thus, to be included in the sample, a study had to report on a comparison between a misinformation and correction condition and a no-misinformation control group. Studies that focused on correction of misinformation without including a no misinformation condition were excluded (e.g., Johnson \& Seifert, 1994). Moreover, the meta-analysis examined processing conflicting information, such that information is first presented as true but is subsequently revoked or challenged. Hence, the sample does not 
include studies in which the misinformation is only presented in the context of negation of this information (e.g., false information is accompanied with the label “myth”, Skurnik, et al., 2005). Also excluded are studies in which misinformation is presented following a forewarning without a post-misinformation correction (e.g., Ecker, Lewandowsky, \& Tang, 2010). Finally, only studies that reported quantitative outcomes using appropriate statistics for calculating an effect size were included in the analyses. When such information was not directly available in the research report, corresponding authors were contacted and the missing information was obtained (Figure 1 illustrates a flow diagram showing the study screening process).

\section{--- Figure 1 ---}

Sample. The final sample included 32 individual studies from 21 research reports (3, 14.3\% unpublished), with a total sample size of 6,527 $(M=203.97, \mathrm{Med}=107, S D=221.05)$. The most common topic of misinformation among the studies that met the inclusion criteria was politics $(k=11)$, followed by health/science $(k=8)$, crime/accidents $(k=7)$, and other $(k=6)$.

Coding. The articles were coded by two coders with a $20 \%$ overlap to ensure continued reliability. Krippendorff's alpha for all variables was satisfactory (range: .71 - 1.0). The relatively low reliability resulted from judgments made by coders examining the original research stimuli, such as establishing the credibility of the information or whether the information carries positive or negative valance.

\section{Moderators}

Coherence of misinformation and correction. The stimuli employed by the researchers of the original studies have been obtained and coded for coherence. Coherence was coded as a binary (high/low) variable based on the amount of detail and context provided in the message. For corrective information to be coded as high in coherence, the message had to mention a 
reason for the distribution of the misinformation or provide an alternative explanation, rather than merely retract the misinformation. Overall, nine effect sizes were associated with highcoherence of misinformation and 13 were coded as low-coherence of misinformation. Further, 14 effect sizes were recorded for high-coherence corrections and 17 effect sizes were recorded for low-coherence corrections.

Time delay. Time delay between misinformation and correction. Each study was coded as either presenting the correction immediately following the misinformation $(k=17)$ or after a filler task $(k=10)$. In five studies, corrections were presented both immediately after the misinformation and after a filler task.

Repetition of misinformation and correction. The number of separate instances in which misinformation has been repeated prior to correction was coded as a continuous variable. Though the vast majority of effect sizes were associated with a single exposure to misinformation, there were some cases where misinformation was repeated twice $(k=6)$ and even three times $(k=2)$. Similarly, the number of time the corrective message has been presented was coded, resulting in 29 effect sizes for a single exposure and three cases in which corrections were presented three times.

Repetition of misinformation in correction. Each corrective message was coded as either reiterating the misinformation $(k=13)$ or only providing the correct information without restating the false information $(k=11)$.

Consistency with worldview. This variable was coded only for studies that manipulated consistency between the participants' worldview and the valance of the correction. For example, if a correction message about a Democrat candidate (e.g., she resigned not due to the previously published allegations of corruption but rather to spend more time with her family) was presented 
to a sample of self-identified Democrats. In total, the sample of studies included six studies with pro-attitudinal corrections and seven studies with counter-attitudinal corrections.

Context. The context of the misinformation was coded as either real-world situations (e.g., pertaining to actual events such as the war in Iraq) $(k=14)$ or fictional events (e.g., a fictitious news story about a rubbery) $(k=18)$.

Source credibility. The source of the misinformation was coded as either having high credibility $(k=7)$ or low credibility $(k=21)$ based on the implied expertise and trustworthiness of the source. Similarly, the source of the corrective message was coded as either having high $(k$ $=15)$ or low credibility $(k=15)$.

Source equivalence. For each study it was determined whether the same source provided both the misinformation and correction $(k=22)$ or whether these two types of information were attributed to two different sources $(k=12)$.

Negativity of misinformation and correction. The valance of the of the misinformation and the correction was determined based on whether the situation is negative (or neutral/positive) assuming the information is correct. Specifically, in 19 cases misinformation was coded as negative and in 13 cases misinformation was coded as natural or positive. In addition, six studies were associated with exposure to negatively valanced corrections and 25 studies exposed participants to positively valanced or neutral corrections.

Linguistic negation. Based on inspection of the stimuli used in the original studies, each correction message was coded for whether it used negation (repeating the misinformation with a negation tag “no”) $(k=13)$ or used affirmative language $(k=9)$. Please see Table 1 for a complete list of coded studies and moderators. 


\section{Analysis}

Comprehensive Meta-Analysis (v.3; Borenstein, Hedges, Higgins, \& Rothstein, 2005) was used to analyze the data, with weights being assigned to primary studies based on the inverse of the variance, as opposed to alternative approaches that assign weights based on sample size. The results focus on random-effects models, allowing to generalize the findings beyond the specific populations from which the data was drawn (Hedges \& Vevea, 1998). For ease of interpretation, we used a correlation coefficient $r$ as the effect size estimate. This coefficient represents the effect of exposure to misinformation that is later corrected compared with a noexposure condition. A negative $r$ indicates stronger continued influence effect, a null effect indicates that the corrective message was able to erase the influence of misinformation, and a positive $r$ suggests that exposure to the corrective message not merely erased the influence of misinformation but also affected participants' beliefs in a direction consistent with the correction.

After analyzing the average effect of continued influence, the analysis focused on the $Q$ statistic to assess the heterogeneity among effect sizes (Borenstein, Hedges, Higgins, \& Rothstein, 2005), defined as:

$$
Q=\sum_{i=1}^{k} W_{i}\left(Y_{i}-M\right)^{2}
$$

Where $W_{i}$ is the study weight $\left(1 / V_{i}\right), Y_{i}$, is the study effect size, $M$ is the summary effect, and $k$ is the number of studies. Thus, the $Q$ statistic computes the deviation of each subgroup from the mean weighted by the inverse-variance to yield the weighted sum of squares (WSS), or Q. Given significant heterogeneity, the potential role played by categorical moderators (i.e., coherence, worldview, context, credibility, equivalence, negativity, and linguistic negation) was 
further probed with $Q$ statistic and the continues moderator (i.e., repetition) was tested with a meta-regression (Rosenthal, 1991).

\section{Results}

Across 32 individual studies, the average continued influence effect on beliefs was weak, negative, and significant ( $r=-.05$, CI [-.10, -.01], $p=.045)$. This suggests that, even after exposure to corrections, some weak traces of misinformation continue to influence people’s beliefs. Further, significant heterogeneity was observed among effect sizes $Q(31)=156.52, p=$ $.0005, I^{2}=80.19$ ), indicating variability that might be explained by moderators (Pearce \& Field, 2015).

Contrary to H1a, there was no significant difference in effect sizes between high $(r=$ .08 , CI $[-.18, .01], p=.09)$ and low-coherence $(r=-.03$, CI $[-.11, .06], p=.54)$ misinformation; $(Q(1)=0.79, p=.37)$. Though-coherence of the misinformation was a nonsignificant moderator, coherence of the correction emerged as a significant predictor of effect sizes; $Q(1)=4.46, p=$ .027, with high-coherence corrections resulting in weaker and nonsignificant continued influence effects $(r=-.03$, CI $[-.12, .06], p=.37)$, compared with corrections low in coherence $(r=-.12$, CI [-.19, -.03], $p=.004)$. Further, the interaction between the coherence of the misinformation and the coherence of the correction was tested by creating four groups that included all possible combinations of the coherence moderator (corresponding to high-coherence misinformation + high-coherence correction, high-coherence misinformation + low-coherence correction, lowcoherence misinformation + high-coherence correction, and low-coherence misinformation + low-coherence correction). As Figure 2 illustrates, there was a borderline significant difference between the resulting subgroups $(Q(3)=2.97, p=.07)$, with the strongest impact of misinformation occurring when high-coherence misinformation was corrected with low- 
coherence correction ( $r=-.19$, CI [-.34, -.04], $p=.04)$.

--- Figure 2 ---

A significant effect of time delay between exposure to misinformation and its correction was found $(Q(1)=4.79, p=.045)$. The continued effect of misinformation was stronger when the correction was presented after a filler task $(r=-.13$, CI [-.28, -.02], $p=.045)$ compared to misinformation that was corrected immediately $(r=.01$, CI $[-.05, .06], p=.87)$. Further, a metaregression was used to test the role played by repetition of misinformation and its correction. In particular, the analysis indicated that repetition of misinformation is a significant predictor of the continued influence effect $(b=-.30, \mathrm{SE}=.12, p=.001)$, with each additional repetition of misinformation enhancing its continued influence on beliefs by an average of .30 points. Though the moderation pattern was in the expected direction, there was no significant difference $(Q(1)=$ $.1 .79, p=.18)$ between studies that repeated the misinformation within the correction $(r=-.10$, CI [-.19, -.01], $p=.03$ ) and studies that used corrective messages without restating the misinformation ( $r=-.02$, CI [-.09, .05], $p=.52$ ). Regretfully, given the lack of variance in our sample, we were not able to test the role played by repetition of corrective information.

As predicted, counter-attitudinal corrections led to stronger continued influence effects ( $r$ $=-.20$, CI [-.32, -.07], $p=.002)$, compared with pro-attitudinal corrections that tended to yield nonsignificant effects ( $r=-.01$, CI $[-.15, .15], p=.72) ; Q(1)=4.78, p=.018$. Additionally, there was no significant difference between real world contexts $(r=-.02$, CI [-.09, .05], $p=.50)$ and constructed, or fictional ( $r=-.04$, CI $[-.13, .04], p=.32)$, contexts; $Q(1)=0.12, p=.73$. The same results were obtained when discrete study topics were considered rather than comparing real world and fictional contexts overall. No significant difference emerged in effects sizes by the topic of misinformation in the study; $Q(2)=0.13, p=.93$; politics $(r=-.04$, CI [-.09, .01], $p$ 
$=.15)$, health/science $(r=-.02$, CI [-.13, .08], $p=.68)$, and crime/accidents $(r=-.07$, CI [-.30, $.18], p=.61)$.

With respect to source credibility, the continued influence effect was stronger $(Q(1)=$ 4.54, $p=.033)$ when misinformation was attributed to a high credibility source $(r=-.18$, CI [$.33,-.02], p=.03)$ compared to a low credibility source $(r=.01$, CI $[-.05, .06], p=.76)$. Yet, the credibility of the source delivering the correction was less important, as there was no significant difference $(Q(1)=3.12, p=.077)$ between high credibility sources $(r=-.09$, CI [-.16, -.02], $p=$ $.02)$ and low credibility sources $(r=.02$, CI $[-.08, .11], p=.70)$. Additionally, there was a significant difference between effect sizes of studies where the source of the misinformation was the same as the source of the correction, as opposed to cases where the source of misinformation was different from the source of correction $(Q(1)=3.74, p=.05)$. As hypothesized, the continued influence effect was weaker when the same source delivered both the correction and the misinformation ( $r=.01$, CI $[-.06, .08], p=.74)$ and stronger effects were observed when different sources were responsible for misinformation and its correction $(r=-.10$, CI [-.18, -.01], $p=.02)$.

Further, there was no significant difference in the continued influence effect $(Q(1)=0.04$, $p=.85)$ between negatively valanced misinformation $(r=-.04$, CI [-.11 .03], $p=.25)$ and neutral or positive misinformation ( $r=-.06$, CI $[-.15, .04], p=.26)$. Similarly, there was no significant difference between the impact of misinformation $(Q(1)=0.99, p=.32$ ) when participants were exposed to negatively valanced $(r=.01$, CI [-.12 .14], $p=.87$ ) corrections and neutral or positive corrections ( $r=-.06$, CI [-.12, -.001], $p=.05)$. Finally, in contrast with our expectation, the continued influence of misinformation did not differ $(Q(1)=0.90, p=.34$ ) by whether the correction message involved linguistic negation ( $r=-.08$, CI [-.12 .01], $p=.04)$ or affirmative 
language $(r=-.08$, CI [-.16, .05], $p=.47)$.

\section{Publication bias}

In order to examine a possible “file drawer” problem, a fail-safe $N$ was calculated (Rosenthal, 1991). According to the results, it would require 73 missing studies with an effect size of 0 to render the average effect size of continuous influence nonsignificant. To this end, though the results are not directly compromised by unpublished research, it can also be argued that the continued influence effect is far from being pervasive and robust.

\section{Discussion}

The current meta-analysis synthesizes four decades of research on continued influence of misinformation. Overall, correction of misinformation does not entirely revert people’s attitudes and beliefs to their baseline levels. Rather, misinformation continues to have a small, albeit significant, effect. The meta-analysis considered six complementary theoretical explanations of this effect, examining features of the misinformation and its correction. A summary of these findings appears in Table 2. Data suggest that repetition of the misinformation, coherence of misinformation coupled with a low-coherence correction, and credibility of the misinformation result in the greatest continued misinformation effect. Conversely, this effect is diminished when correction is delivered immediately, when it is consistent with the message recipient's worldview, and it is attributed to the same source that was responsible for the misinformation. Together, these results provide at least partial support to explanations relying on theories of mental models, fluency, world-view consistent elaboration and source evaluation. No support was found for the negativity bias and retrieval failure according to the schema plus tag theory.

Considering the pattern of results (see Table 2), mental models appears to be the most 
consistently supported explanation. Curiously, it also seems that the encoding of misinformation rather than the correction plays a more major role: the repetition of misinformation creates fluency (but not debunking efforts), and the credibility of the source of the misinformation (but not the correction) matters. While reading the misinformation, individuals construct an event model, namely a mental representation of a specific situation. During the reading process, as the narrative evolves, individuals continuously update the model (Zawaan, Langston, Graesser, 1995). Thus, when the correction appears alongside the misinformation (e.g., a balanced news story containing both sides of the argument - the misinformation and the rebuttal) readers are able to encode the corrective information and incorporate it in the mental model displacing the misinformation. However, when the information is not corrected immediately, the episodic model generated while reading the misinformation is already integrated into the schematic representation of the issue. As noted in past research, "the initial integration of information when the model is being built is more readily performed than is its updating after a retraction” (Ozturk, Li, \& Sakamoto, 2015, p. 2407).

The significant findings concerning world view-consistency can also be interpreted in light of theories of mental representation. Political ideology constitutes a cognitive schema whose epistemological function includes explaining phenomena and making predictions (Jost, Federico, \& Napier, 2009). In general, schemas guide attention to information, help organize and store it, fill-in gaps, and assist in problem solving (Busselle, 2017). Like other types of schema, political ideology guides individuals’ attention to information. Thus, with counterattitudinal misinformation, the message recipient is motivated to attend to a worldviewconsistent correction that will reduce the cognitive dissonance caused by the misinformation. Once the individual attends to the world-view consistent corrective information, ideology can be 
used as a schema to fill-in the missing information. For example, consider a situation in which the correction is low in coherence but is worldview-consistent. In other words, it negates the misinformation without providing an alternative explanation, but it is consistent with the message recipient's beliefs. The message recipient then will be motivated to generate a worldview-driven explanation. It is, therefore, plausible that worldview-consistency increases tolerance for low-coherence correction, since the gaps in the mental model can be supplemented by the overarching schema.

Whereas multiple findings in the meta-analysis are consistent with predictions derived from theories of mental models, no support was found for negativity bias and linguistic negation. Negation language and the valance of the misinformation/ correction did not seem to moderate the persistence of misinformation. However, no studies in the sample specifically manipulated these effects. Future research will be well served to examine negativity bias and negation more systematically by manipulating these variables within the study design.

\section{Limitations}

Though meta-analyses offer a bird's view of the literature that cannot be attained by individual studies, they are also limited to the pool of available studies. Consequently, some potential moderators and relevant outcomes were not analyzed due to insufficient number of cases. For example, there is a need for additional research on repetitions of correction messages as only few studies manipulated this variable in a systematic way. Moreover, the meta-analysis exclusively focused on beliefs as an outcome because of the limited number of studies reporting other types of outcomes. It is not implausible that the continued influence of misinformation has different effects on recall, knowledge, or behavior, as it has on beliefs. Arguably, these outcomes represent different stages within the process of persuasion (or debiasing) (McGuire, 
1968) and they can be affected in different ways.

Finally, the current meta-analysis was limited to randomized experiments which inherently suggests a tradeoff between internal and external validity. Although the current study's approach offers a stronger theoretical understanding of how misinformation effects can persist, it is important to be cautious when generalizing these findings to real-world settings. Specifically, randomized experiments ensure equivalence between study participants who are exposed to the misinformation and correction and those who were exposed to neither. However, outside the artificially controlled experimental environment, individuals who are exposed to misinformation are likely to differ on various background characteristics from those who were not exposed to the misinformation at all (e.g., due to processes of selective exposure individuals are inclined to seek out attitude-confirming information). Hence, while the current study asserted that correction of misinformation has the potential to bring one's attitude to the same level as that of individuals who were never exposed to the misinformation in the first place, the effects uncovered in this meta-analysis represent a best-case scenario.

\section{Practical Implications}

The results of the meta-analysis can be used to prescribe specific recommendations for more effective misinformation correction efforts.

Credibility attack. Corrections should criticize the credibility of the source of the misinformation. This serves two functions. First, source credibility is central to processing of the initial (mis)information but not for the correction source. Thus, trying to undo the damage done by climate change deniers and vaccine skeptics with messages that rely, primarily, on the expertise of their sources is likely to be futile. Instead, the correction should focus on discrediting the sources of misinformation. For example, rather than emphasizing the knowledge 
of a climate science expert, messages should highlight the lack of expertise and relevant training of climate change skeptics. Second, questioning the credibility of the misinformation source can enhance the coherence of the corrective message. Put differently, discrediting the source as biased and lacking good will can offer an explanation for the spread of the misinformation and make it easier for message consumers to maintain a coherent mental model that dismisses the misinformation.

Same-source correction. Corrections coming from the same source as the misinformation carry more weight than messages delivered by different sources. This finding endorses the FTC mandate for corrective information to be attributed to the same source as the original misinformation. By extension, corrective campaigns can choose spokespersons who are members from the same social group as the source of misinformation. For instance, to effectively discredit an anti-vaccination campaign led by parents of children with autism, it may be helpful to feature parents of children with autism who support vaccines.

World-view consistency. Corrections work better when they fit into people's worldview and when misinformation runs counter to preexisting beliefs. Thus, designers of corrective messages should strive to emphasize how the accurate information fits with people’s broader belief system. This approach can be extremely challenging, especially when dealing with valueladen beliefs (e.g., political ideology); however, in some cases, it is likely to work. For instance, consumers often fear genetically modified organisms (GMOs) because, supposedly, "big food” companies are trying to take advantage of unsuspecting individuals. Specifically, fear of GMOs is rooted in false notions that moving genes between species is unnatural and risky; yet, these erroneous beliefs might sound very convincing because they align with people’s general fears about health and science (Pew Research Center, 2016). A more promising dialogue with GMOs 
skeptics would emphasize its positive impact on farmers and the economy, rather than focusing, almost exclusively, on the technology and science behind it (Ben-Shahar, 2017).

Contextualization and explanation. Finally, corrective messages need to provide not only a negation of the misinformation but also explanations that have both internal (i.e., compelling and consistent account) and external (i.e., fits the general understanding of how the world operates) coherence. This is especially critical when the initial misinformation provides a complete and clear account. For example, instead of emphasizing agreement among scientists, a much more compelling story about the root of climate science denial can recount how oil companies sponsor political lobbyists to protect their profits and limit future liabilities. This is a simple story that fits within laypeople’s preexisting understanding of the problematic relationship between politicians and interest groups.

In sum, the results of the study paint an optimistic outlook on the effectiveness of correction. Although there is an undeniable effect of misinformation in the face of correction, it is far from being the robust and irreversible phenomenon it is often believed to be. Therefore, researchers and practitioners can utilize various message design characteristics to avert or, at least, minimize the threat of misinformation. It is our hope that this meta-analysis will contribute to the existing body of knowledge and prove useful in correcting misinformation. 


\section{References}

*References for studies included in the meta-analysis are provided in Appendix A.

Allen, M., Preiss, R. W., \& Gayle, B. M. (2006). Meta-analytic examination of the base-rate fallacy. Communication Research Reports, 23, 45-51. doi:10.1080/17464090500535863

Armstrong, G.M., Gurol, M., Russ, F.A. (1979). Detecting and correcting deceptive advertising. Journal of Consumer Research, 6, 237-246. doi:10.1086/208765

Barbetta, P.M., Heward, W. L., Bradley, D. M., \& Miller, A. D. (1994). Effects of immediate and delayed error correction on the acquisition and maintenance of sight words by students with developmental disabilities. Journal of Applied Behavior Analysis, 27, 177178. doi:10.1901/jaba.1994.27-177

Baumeister, R.F., Bratslavsky, E., Finkenauer, C., \& Vohs, K. D. (2001). Bad is stronger than good. Review of General Psychology, 5, 323-370. doi:10.1037/1089-2680.5.4.323

Bebbington, K., MacLeod, C., Ellison, T. M., \& Fay, N. (2017). The sky is falling: Evidence of a negativity bias in the social transmission of information. Evolution and Human Behavior, 38, 92-101. doi:10.1016/j.evolhumbehav.2016.07.004

Ben-Shahar, O. (2017). Consumers fear GMOs because 'Big Food’ companies bombard them with misinformation. Genetic Literacy Project. Retrieved from: https://geneticliteracyproject.org/2017/12/18/viewpoint-consumers-fear-gmos-big-foodcompanies-bombard-misinformation/

Berinsky, A. J. (2017). Rumors and health care reform: Experiments in political misinformation. British Journal of Political Science, 47, 241-262. doi:10.1017/S0007123415000186

Blank, H., \& Launay, C. (2014). How to protect eyewitness memory against the misinformation effect: A meta-analysis of post-warning studies. Journal of Applied Research in Memory 
and Cognition, 3, 77-88. doi:10.1016/j.jarmac.2014.03.005

Borenstein, M., Hedges, L., Higgins, J., \& Rothstein, H. (2005). Comprehensive meta-analysis: A computer program for meta-analysis [Computer software]. Englewood, NJ: Biostat Inc.

Busselle, R. (2017). Schema theory and mental models. In: P. Rossler (Ed.), The International Encyclopedia of Media Effects, (pp. 1753-1760) Wiley Blackwell.

Cappella, J. N., Maloney, E., Ophir, Y., \& Brennan, E. (2015). Interventions to correct misinformation about tobacco products. Tobacco regulatory science, 1, 186-197. doi:10.18001/TRS.1.2.8

Chan, M. P. S., Jones, C. R., Hall Jamieson, K., \& Albarracín, D. (2017). Debunking: A metaanalysis of the psychological efficacy of messages countering misinformation. Psychological Science, 28, 1531-1546. doi:10.1177/0956797617714579

Cho, C. H., Martens, M. L., Kim, H., \& Rodrigue, M. (2011). Astroturfing global warming: It isn't always greener on the other side of the fence. Journal of Business Ethics, 104, 571587. doi:10.1007/s10551-011-0950-6

Clark, H. H., \& Chase, W. G. (1972). On the process of comparing sentences against pictures. Cognitive psychology, 3, 472-517. doi:10.1016/0010-0285(72)90019-9

Duval, S., \& Tweedie, R. (2000). Trim and fill: A simple funnel-plot-based method of testing and adjusting for publication bias in meta-analysis. Biometrics, 56, 455-463. doi:10.1111/j.0006-341X.2000.00455.X

Ecker, U. K., Lewandowsky, S., Cheung, C. S., \& Maybery, M. T. (2015). He did it! She did it! No, she did not! Multiple causal explanations and the continued influence of misinformation. Journal of Memory and Language, 85, 101-115.

Ecker, U. K., Lewandowsky, S., Swire, B., \& Chang, D. (2011). Correcting false information in 
memory: Manipulating the strength of misinformation encoding and its retraction. Psychonomic Bulletin \& Review, 18, 570-578. doi:10.3758/s13423-011-0065-1

Egger, M., Smith, G. D., Schneider, M., \& Minder, C. (1997). Bias in meta-analysis detected by a simple, graphical test. BMJ, 315, 629-634. doi:10.1136/bmj.315.7109.629

Festinger, L. (1954). A theory of social comparison processes. Human relations, 7 117-140. doi:10.1177/001872675400700202

Fiedler, K., Walther, E., Armbruster, T., Fay, D., \& Naumann, U. (1996). Do you really know what you have seen? Intrusion errors and presuppositions effects on constructive memory. Journal of Experimental Social Psychology, 32, 484-511. doi:10.1006/jesp.1996.0022

Flynn, D. J., Nyhan, B., \& Reifler, J. (2017). The nature and origins of misperceptions: Understanding false and unsupported beliefs about politics. Political Psychology, 38, 127-150. doi:10.1111/pops.12394

Foster, J. L., Huthwaite, T., Yesberg, J. A., Garry, M., \& Loftus, E. F. (2012). Repetition, not number of sources, increases both susceptibility to misinformation and confidence in the accuracy of eyewitnesses. Acta Psychologica, 139, 320-326.

doi:10.1016/j.actpsy.2011.12.004

Guess, A., Nyhan, B., \& Reifler, J. (2018). Selective Exposure to Misinformation: Evidence from the consumption of fake news during the 2016 US presidential campaign. European Research Council.

Guillory, J. J., \& Geraci, L. (2013). Correcting erroneous inferences in memory: The role of source credibility. Journal of Applied Research in Memory and Cognition, 2, 201-209. doi:10.1016/j.jarmac.2013.10.001 
Gwaltney, C. J., Metrik, J., Kahler, C. W., \& Shiffman, S. (2009). Self-efficacy and smoking cessation: A meta-analysis. Psychology of Addictive Behaviors, 23, 56-66. doi:10.1037/a0013529

Hansen, J., \& Wänke, M. (2009). Liking what's familiar: The importance of unconscious familiarity in the mere-exposure effect. Social Cognition, 27, 161-182. doi:10.1521/soco.2009.27.2.161

Hedges, L. V., \& Vevea, J. L. (1998). Fixed-and random-effects models in meta-analysis.

Psychological Methods, 3, 486-504. doi:10.1037/1082-989X.3.4.486

Ito, T. A., Larsen, J. T., Smith, N. K., \& Cacioppo, J. T. (1998). Negative information weighs more heavily on the brain: The negativity bias in evaluative categorizations. Journal of Personality and Social Psychology, 75, 887-900. doi:10.1037/0022-3514.75.4.887

Johnson, H. M., \& Seifert, C. M. (1994). Sources of the continued influence effect Journal of Experimental Psychology: Learning, Memory, and Cognition, 20, 1420-1436. doi:10.1037/0278-7393.20.6.1420

Jost, J. T., Federico, C. M., \& Napier, J. L. (2009). Political ideology: Its structure, functions, and elective affinities. Annual Review of Psychology, 60, 307-337. doi:10.1146/annurev.psych.60.110707.163600

Lemon, D. (2018, April 12). CNN Tonight. CNN. Retrieved from: https://www.alternet.org/cnnsdon-lemon-shuts-down-ex-trump-aide-live-air

Lewandowsky, S., Ecker, U. K. H., Seifert, C. M., Schwarz, N., \& Cook, J. (2012).

Misinformation and its correction: Continued influence and successful debiasing.

Psychological Science in the Public Interest, 13, 106-131. doi:10.1177/1529100612451018

Mayo, R., Schul, Y., \& Burnstein, E. (2004). “I am not guilty” vs “I am innocent”: Successful negation may depend on the schema used for its encoding. Journal of Experimental 
Social Psychology, 40, 433-449. doi:10.1016/j.jesp.2003.07.008

Mazis, M. B., \& Adkinson, J. E. (1976). An experimental evaluation of a proposed corrective advertising remedy. Journal of Marketing Research, 13, 178-183. doi:10.2307/3150855

McGuire, W. J. (1968). Personality and attitude change: An information processing theory. In A. G. Greenwald, T. C. Brock, \& T. M. Ostrom (Eds.), Psychological foundations of attitudes (pp. 171-196). New York, NY: Academic Press.

Nyhan, B., \& Reifler, J. (2012). Misinformation and corrections: Research findings from social science. Unpublished manuscript, Dartmouth College, Hanover, NH.

Nyhan, B., \& Reifler, J. (2015). Displacing misinformation about events: An experimental test of causal corrections. Journal of Experimental Political Science, 2, 81-93. doi:10.1017/XPS.2014.22

Nyhan, B., Reifler, J., \& Ubel, P. A. (2013). The hazards of correcting myths about health care reform. Medical Care, 51, 127-132. doi:10.1097/MLR.0b013e318279486b

O’Keefe, D. J. (2015). Message generalizations that support evidence-based persuasive message design: Specifying the evidentiary requirements. Health Communication, 30, 106-113. doi:10.1080/10410236.2014.974123

Ozturk, P., Li, H., \& Sakamoto, Y. (2015, January). Combating rumor spread on social media: The effectiveness of refutation and warning. In System Sciences (HICSS), 2015 48th Hawaii International Conference on (pp. 2406-2414).

Petty, R. E., \& Cacioppo, J. T. (1986). Communication and persuasion (pp. 1-24). NY: Springer.

Reber, R., \& Schwarz, N. (1999). Effects of perceptual fluency on judgments of truth. Consciousness and Cognition, 8, 338-342. doi:10.1006/ccog.1999.0386

Rosenthal, R. (1991). Meta-analytic procedures for social research (2nd ed.). Beverly Hills, CA: Sage. 
Rozin, P., \& Royzman, E. B. (2001). Negativity bias, negativity dominance, and contagion. Personality and Social Psychology Review, 5, 296-320. doi:10.1207/S15327957PSPR0504_2

Schwarz, N., Sanna, L. J., Skurnik, I., \& Yoon, C. (2007). Metacognitive experiences and the intricacies of setting people straight. Advances in Experimental Social Psychology, 39, 127-161. doi:10.1016/S0065-2601(06)39003-X

Schwarz, N. (2015). Metacognition. In M. Mikulincer, P. R. Shaver, E. Borgida, \& J. A. Bargh (Eds.), APA handbook of personality and social psychology: Attitudes and social cognition (pp. 203-229). Washington, DC: American Psychological Association.

Skurnik, I., Yoon, C., \& Schwarz, N. (2007). “Myths \& Facts” about the flu: Health education campaigns can reduce vaccination intentions. Unpublished manuscript.

Vosoughi, S., Roy, D., \& Aral, S. (2018). The spread of true and false news online. Science, 359, 1146-1151. doi:10.1126/science.aap9559

Walter, N., \& Murphy, S. T. (2018). How to unring the bell: A meta-analytic approach to correction of misinformation. Communication Monographs, advance online publication doi:10.1080/03637751.2018.1467564

Zillmann, D. (2000). Exemplification theory of media influence. In J. Bryant, D. Zillmann, J. Bryant, M. B. Oliver (Eds.), Media effects (pp. 19-42), Lawrence Erlbaum Associates: Mahwah, NJ.

Zwaan, R. A., Langston, M. C., \& Graesser, A. C. (1995). The construction of situation models in narrative comprehension: An event-indexing model. Psychological science, 6, 292297. doi:10.1111/j.1467-9280.1995.tb00513.x 
Table 1. Studies included in Meta-Analysis

\begin{tabular}{|c|c|c|c|c|}
\hline Study & Year & Study & $N$ & Topic \\
\hline Aikin et al. ${ }^{\mathrm{cg}}$ & 2015 & 1 & 504 & Misleading direct-to-consumer drug ads \\
\hline Armstrong et al. ${ }^{\text {bcegh }}$ & 1979 & 1 & 102 & Deceptive ads for Listerine mouthwash \\
\hline Berinsky ${ }^{\text {bdefh }}$ & 2015 & 1 & 556 & Rumors about healthcare reform \\
\hline Cobb, Nyhan, \& Reifler ${ }^{\mathrm{C}}$ & 2013 & 1 & 168 & Misleading information about a politician \\
\hline Dixon et al. ${ }^{\text {bfh }}$ & 2015 & 1 & 371 & False information about the autism-vaccine link \\
\hline Ecker et al. ${ }^{\text {abdhij }}$ & 2014 & 1 & 96 & Fictitious report about a liquor store robbery \\
\hline Ecker et al. ${ }^{\text {abefh }}$ & 2011 & 1 & 115 & Fictitious news report about a warehouse fire \\
\hline Greitemeyer et al. ${ }^{\mathrm{cf}}$ & 2015 & 1 & 124 & Scientific retraction \\
\hline Greitemeyer et al. ${ }^{\mathrm{cf}}$ & 2015 & 2 & 180 & Scientific retraction \\
\hline Greitemeyer ${ }^{\mathrm{g}}$ & 2014 & 1 & 106 & Scientific retraction \\
\hline Huang defh & 2017 & 1 & 252 & Various rumors \\
\hline Huang befh & 2017 & 2 & 248 & Various rumors \\
\hline Mazis \& Adkinson ${ }^{\mathrm{bcg}}$ & 1976 & 1 & 54 & Deceptive ads for a mouthwash brand \\
\hline Misra $^{\text {abce }}$ & 1992 & 1 & 108 & Deceptive information regarding ability \\
\hline Misra ${ }^{\text {abcef }}$ & 1992 & 2 & 51 & Rumors about McDonald's use of worm meat \\
\hline Misra $^{\text {abce }}$ & 1992 & 3 & 48 & Misleading information regarding supermarkets \\
\hline Nyhan \& Reifler ${ }^{\text {bdefh }}$ & 2015 & 1 & 750 & Rumors about political misconduct \\
\hline Rapp \& Kendeouce & 2007 & 1 & 48 & Refuted story \\
\hline Rich \& Zaragoza $a^{\text {abcdf }}$ & 2016 & 1 & 111 & Deceptive information regarding a jewelry theft \\
\hline Rich \& Zaragoza ${ }^{\text {abcdef }}$ & 2016 & 2 & 99 & Deceptive information regarding a jewelry theft \\
\hline Sawyer \& Semenik ${ }^{\mathrm{gl}}$ & 1978 & 1 & 142 & Deceptive ads for Listerine mouthwash \\
\hline Thorson ${ }^{\text {cfhlij }}$ & 2013 & 3a & 87 & Misleading information about misconduct \\
\hline Thorson $^{\text {cfhlij }}$ & 2013 & $3 b$ & 92 & Misleading information about health care policy \\
\hline Thorson ${ }^{\text {cfhlij }}$ & 2013 & 3c & 81 & Misleading information about misconduct \\
\hline Thorson $^{\text {cdefj }}$ & 2016 & 1 & 96 & Misleading information about misconduct \\
\hline Thorson ${ }^{\text {bcdfhij }}$ & 2016 & 2 & 316 & Misleading information about misconduct \\
\hline Thorson ${ }^{\text {cfhij }}$ & 2016 & 3 & 307 & Misleading information about misconduct \\
\hline Wood bdegl & 2014 & 3 & 1012 & Misinformation regarding climate science \\
\hline Wyer \& Budesheim ${ }^{c f}$ & 1987 & 1 & 69 & Misleading information about a person \\
\hline Wyer \& Budesheim ${ }^{\mathrm{c}}$ & 1987 & 2 & 69 & Misleading information about a person \\
\hline Wyer \& Budesheim ${ }^{\mathrm{C}}$ & 1987 & 3 & 69 & Misleading information about a person \\
\hline Wyer \& Unverzagt ${ }^{\text {cef }}$ & 1985 & 1 & 96 & Misleading information about a person \\
\hline
\end{tabular}

Note. ${ }^{\mathrm{a}}$ credible misinformation; ${ }^{\mathrm{b}}$ credible correction; ${ }^{\mathrm{c}}$ same source; ${ }^{\mathrm{d}}$ high-coherence

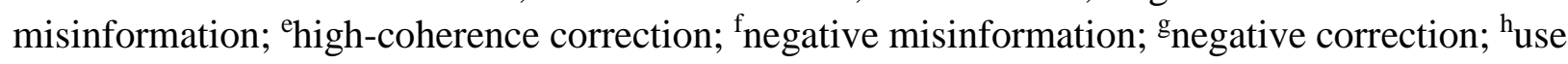
negation; ${ }^{\mathrm{i}}$ pro-attitudinal correction; ${ }^{\mathrm{j}}$ counter-attitudinal correction; ${ }^{\mathrm{l}}$ unpublished. 
Table 2. Summary of Hypotheses, Variables and Findings

\begin{tabular}{|c|c|c|c|c|}
\hline Theory and $\mathrm{H \#}$ & Variable & Misinformation & Correction & Interaction \\
\hline \multirow[t]{2}{*}{ H1-2: Mental models } & Coherence & n.s. & supported & supported \\
\hline & Time delay & & supported & \\
\hline \multirow[t]{2}{*}{ H3: Fluency } & Repetition & supported & n.s. & \\
\hline & $\begin{array}{l}\text { Repetition of misinformation } \\
\text { in correction }\end{array}$ & & n.s. & \\
\hline \multirow[t]{2}{*}{$\begin{array}{l}\text { H4-H5: Worldview consistent } \\
\text { elaboration }\end{array}$} & $\begin{array}{l}\text { Consistency with preexisting } \\
\text { attitudes }\end{array}$ & $*$ & supported & \\
\hline & Topic constructed/real & n.s. & & \\
\hline \multirow[t]{2}{*}{ H6-H7: Source evaluation } & Credibility & supported & n.s. & \\
\hline & Equivalence & & supported & \\
\hline H8: Negativity bias & Negativity & n.s. & n.s. & \\
\hline H9: Retrieval failure & Linguistic negation & & n.s. & \\
\hline
\end{tabular}

Note. * Impossible to compute due to multicollinearity or unavailability of studies. 
Figure 1. PRISMA flow diagram showing the study screening process

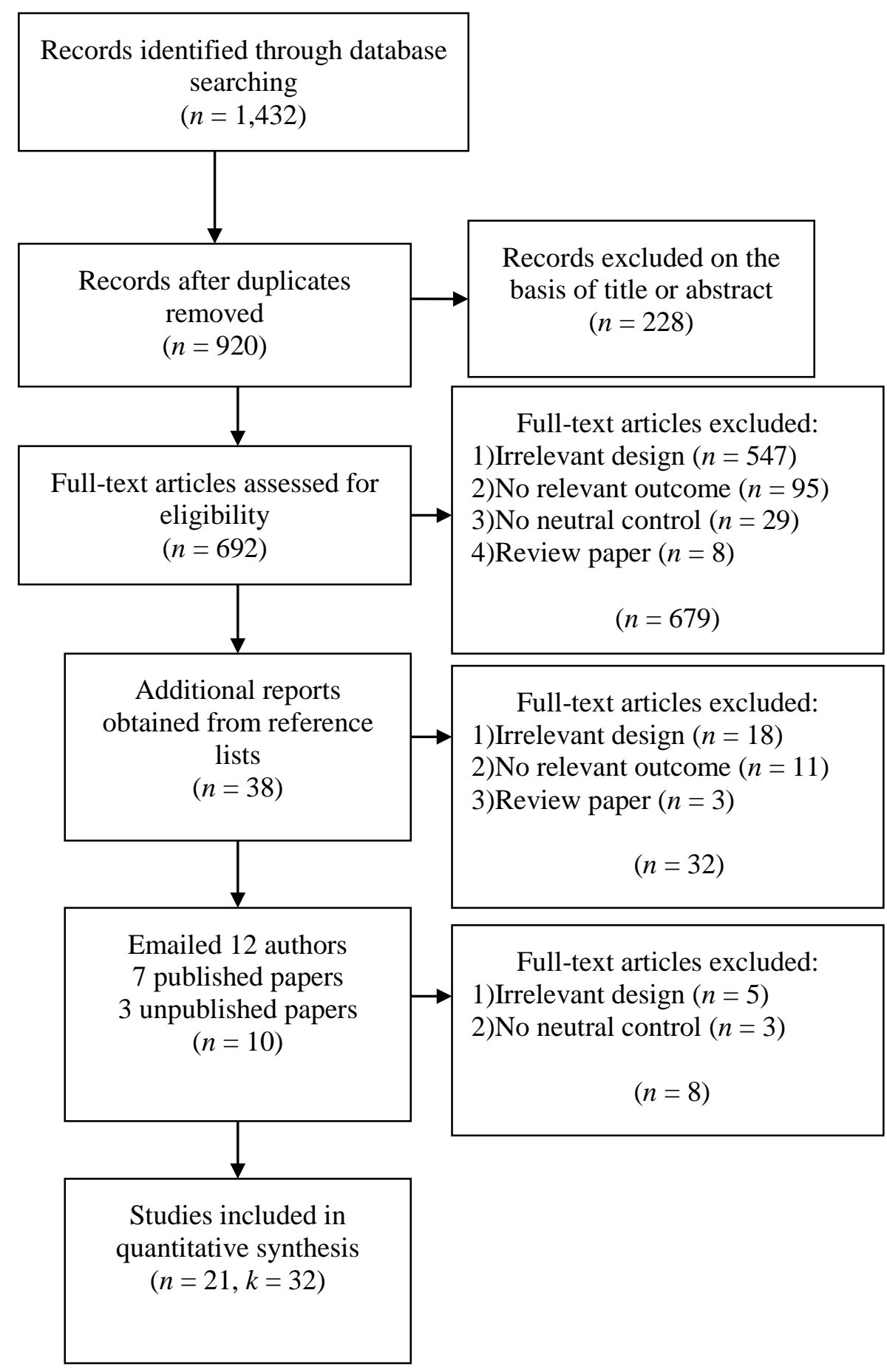


Figure 2. The continued influence effect by coherence of misinformation (high/low) and coherence of correction (high/low)

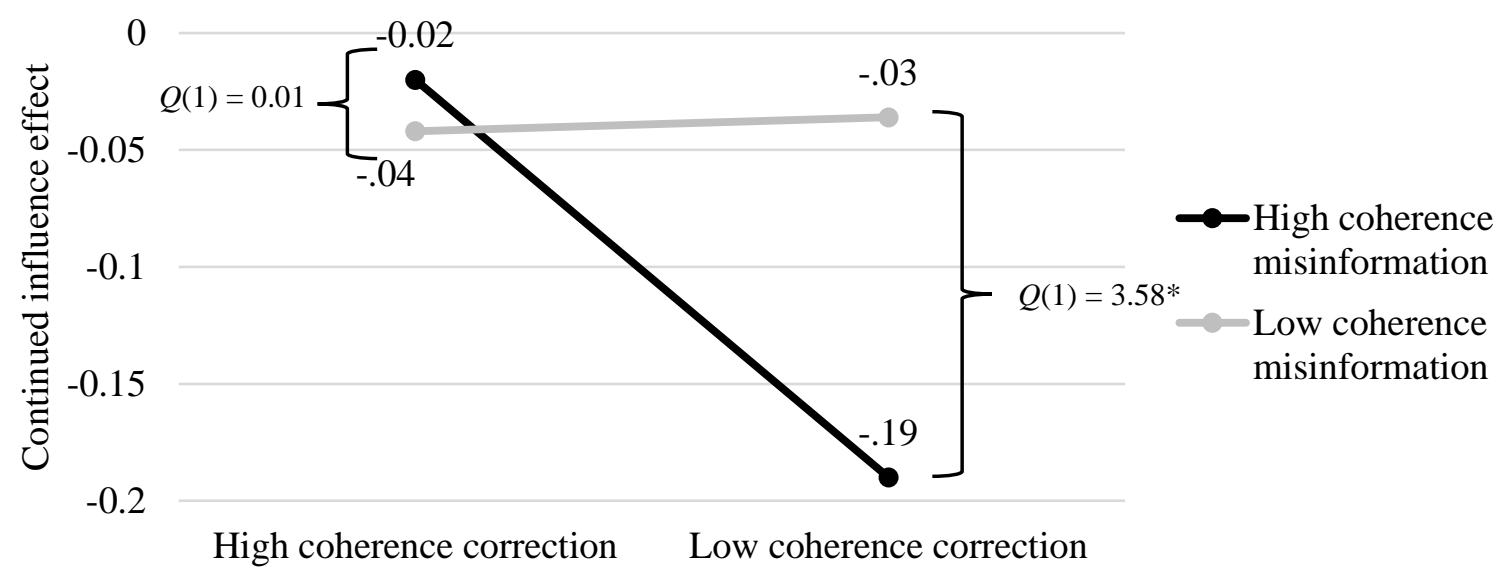

